\title{
ON THE PROBLEM OF MINIMIZING THE VOLUME OF A VIBRATING CANTILEVER*
}

\author{
SOUHAIL EL-ASFOURI, OLIN JOHNSON, AND BAYLISS MCINNIS (University of Houston)
}

1. Introduction. The problem of finding the optimal shape of a transversely oscillating cantilever beam has been recently investigated by Vepa [1]. From the nature of the Hamiltonian, Vepa shows that the problem of minimizing the weight of a cantilever beam with the first fundamental frequency in transverse vibration specified does not possess a solution in the absence of geometric constraints on the design variable. Hence, Vepa imposes a constraint in the form of a lower bound on the design variable which is the mass per unit length of the beam. This form of constraint results in a corner at the end of the portion of uniform radius. The resulting lack of smoothness in the contour raises doubts in regards to the actual physical behavior which can be expected from the beam in an application. In this note we introduce a different approach for introducing this geometric constraint. The reformulated problem yields a smooth contour (i.e., without a corner), but results in a sub-optimal solution. An interesting feature of this work is that the Ritz method in a space of cubic splines was used to find the suboptimal solution.

2. Statement of the problem. For a cantilever beam performing small harmonic transverse vibrations under its own weight, the differential equation for lateral vibrations from [1] is

$$
\left[\alpha^{2}(x) y^{\prime \prime}(x)\right]^{\prime \prime}-\beta \alpha(x) y(x)=0
$$

where $y(x)$ is the lateral deflection in the plane of bending; $\alpha(x)$ is the dimensionless area function, $\alpha(x)=A(x) l / V, V$ is the volume of the beam; $l$ is the length, $\beta=\omega^{2}\left(\rho l^{5} / E c V\right)$ is a dimensionless constant, $c=I / A^{2}$ is a constant characteristic of the cross-section, $\omega$ is the frequency of vibration; and the prime $(')$ denotes the derivative with respect to $x$ [2]. The boundary conditions for a cantilever beam are obtained from physical considerations as follows: At the fixed end $x=0$, the deflection and slope are zero

$$
y(0)=0, \quad y^{\prime}(0)=0 .
$$

At the free end, $x=1$, the moment and the shear are zero

$$
\alpha^{2} y^{\prime \prime}(1)=0, \quad\left(\alpha^{2} y^{\prime \prime}\right)^{\prime}(1)=0
$$

It is required to find the distribution of the area function $\alpha(x)$ of a beam vibrating at a certain natural frequency such that the volume of the beam is minimum. Thus a

* Received January 20, 1975. Supported in part by NSF Grant Number DCR 74-17282. 
minimum is required for

$$
V=\int_{0}^{1} \alpha(x) d x
$$

subject to the differential constraint (1) and the boundary conditions (2).

In order to express the equation of motion (1) as a first-order system of ordinary differential equations, we introduce the vector of state variables $q$ to obtain the system

$$
q^{\prime}=A q ; \quad A=\left[\begin{array}{cccc}
0 & 1 & 0 & 0 \\
0 & 0 & 1 / \alpha^{2} & 0 \\
0 & 0 & 0 & 1 \\
\alpha \beta & 0 & 0 & 0
\end{array}\right]
$$

Forming the Lagrangian $L$ by introducing the vector of Lagrange multipliers $\lambda$ and noting that if all boundary conditions are homogeneous they drop out from the Lagrangian, we have

$$
L=\int_{0}^{1} \alpha(x) d x+\int_{0}^{1}\left\langle\lambda,-q^{\prime}+A q\right\rangle d x
$$

where $L$ is to be minimized [3].

3. Necessary conditions. The necessary conditions for minimizing $L$ are

$$
\begin{aligned}
& \partial L / \partial q=A^{T} \lambda+\lambda^{\prime}=0, \\
& \partial L / \partial \alpha=1+\lambda^{T}(d A / d \alpha) q=0,
\end{aligned}
$$

or

$$
\lambda^{\prime}=-A^{T} \lambda
$$

and

$$
\alpha=\left[\frac{2 \lambda_{2} q_{3}}{1+\beta \lambda_{4} q_{1}}\right]^{1 / 3} .
$$

Eq. (9) yields the optimal solution of the design variable $\alpha(x)$. It can be shown that this solution does not satisfy the additional necessary optimality condition [4] which requires the Hamiltonian to be a constant when evaluated on an extremal trajectory if the terminal of the independent variable is fixed and the Hamiltonian does not depend explicitly on the independent variable. In order to satisfy this condition Vepa [1] imposes a geometric constraint in the form of an inequality constraint on the linear mass density ${ }^{1}$ $\mu(x)$ :

$$
\mu(x)=\mu_{b}, \quad l_{b} \leq x \leq l,
$$

where $l_{b}$ and $\mu_{b}$ are some prescribed values. Another way of imposing a geometric constraint on the design variable $\alpha(x)$ is to perturb the necessary condition (9) and require

${ }^{1}$ Vepa's formulation is in terms of the linear mass density $\mu(x)$ defined by $M=\int_{0}^{1} \mu(x) d x$ where $M$ is the total mass of the beam rather than the area function formulation used here. 
that the area distribution of the beam at the free end to be a finite positive amount rather than zero as would result from (9). One way to introduce this constraint and obtain a sub-optimal solution is to add this lower bound on $\alpha(x)$ to the right-hand side of (9). Assume that $\alpha(x)$ is desired to be equal to $\alpha_{b}{ }^{\prime}$ at the end $x=1$; then the solution for $\alpha(x)$ becomes

$$
\alpha(x)=\left[\frac{2 \lambda_{2} q_{3}}{1+\beta\left(\lambda_{4}\right)\left(q_{1}\right)}+\alpha_{b}\right]^{1 / 3}
$$

where $\alpha_{b}=\left(\alpha^{\prime}{ }_{b}\right)^{3}$. This approach has the advantage of yielding a smooth contour for the beam.

4. Solution by Ritz method in a space of cubic splines. The solution for $\alpha(x)$ in (11) was obtained using the Ritz method $[3,5]$ by first constructing the approximate solution of the Lagrange multiplier $\lambda$ as a linear combination of basis functions $\psi_{i}$, $i=1, \cdots, M$. The basis functions used to obtain the approximate solutions were the patch basis constructed from the space of cubic splines with a uniform mesh of norm $h$ such that $x_{i}=i h, 0 \leq i \leq N$, where $N$ is the number of partitions of the unit interval $[0,1]$. Consequently we can express $\lambda$ as

$$
\lambda(x)=\sum_{i=1}^{M} c_{i} \psi_{i}(x) \quad 0 \leq x \leq 1
$$

where the $c_{i}$ are constants that are selected so that the Lagrangian $L(\lambda)$ is minimal. Once the solution for $\lambda$ is found, then the solution for $q$ and $\alpha(x)$ can be found. The solution for the design variable $\alpha(x)$ is shown in Fig. 1 for different desired values of the area distribution of the beam at the free end.

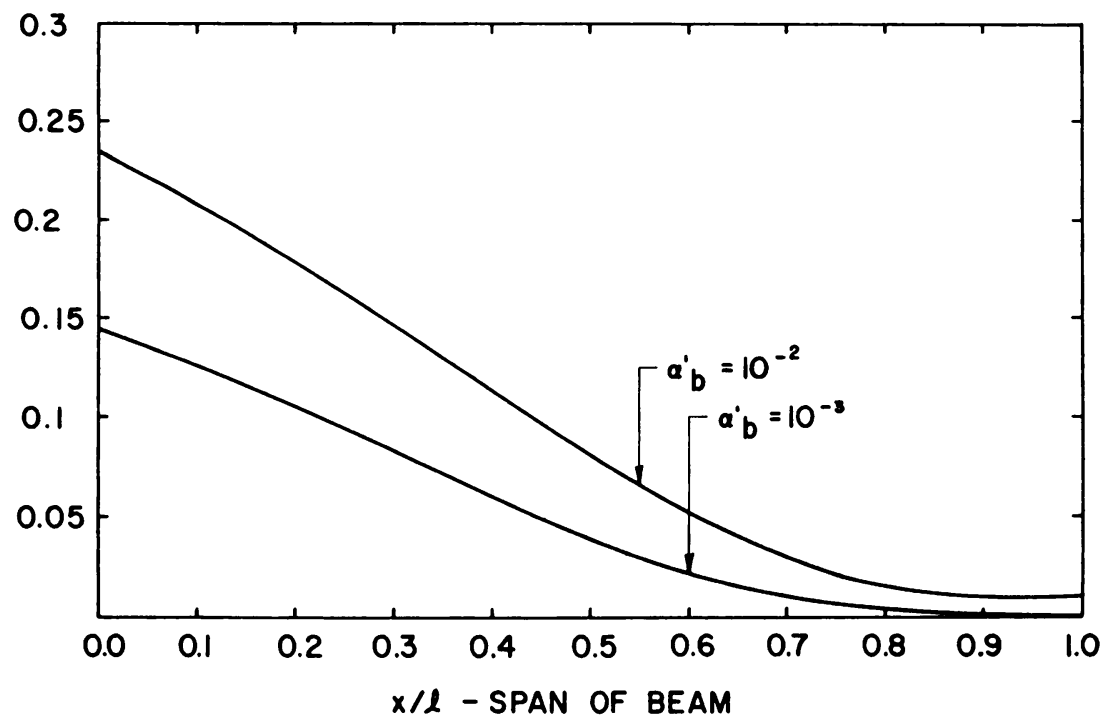

FIG. 1. Sub-optimal cross-sectional area distribution of vibrating beams. 


\section{REFERENCES}

[1] K. Vepa, On the existence of solutions to optimization problems with eigenvalue constraints, Quart. Appl. Math. 31, 329-341 (1973)

[2] F. I. Niordson, The optimal design of a vibrating beam, Quart. Appl. Math. 23, 47-53 (1965)

[3] W. E. Bosarge, Jr. and O. G. Johnson, Error bounds of high order accuracy for the state regulator problem via piecewise polynomial approximations, SIAM J. Control 9, 15-27 (1971)

[4] D. E. Kirk, Optimal control theory, Englewood Cliffs, New Jersey: Prentice Hall, 1970

[5] W. E. Bosarge, Jr. and O. G. Johnson, Numerical propertics of the Ritz-Trefftz algorithm for optimal control, Numerical Mathematics, Communications of the ACM 14, 402-406 (1971) 